\title{
Fractional Fourier optics
}

\author{
Haldun M. Ozaktas \\ Department of Electrical Engineering, Bilkent University, 06533 Bilkent, Ankara, Turkey
}

David Mendlovic

Faculty of Engineering, Tel Aviv University, 69978 Tel Aviv, Israel

Received May 20, 1994; accepted August 22, 1994

There exists a fractional Fourier-transform relation between the amplitude distributions of light on two spherical surfaces of given radii and separation. The propagation of light can be viewed as a process of continual fractional Fourier transformation. As light propagates, its amplitude distribution evolves through fractional transforms of increasing order. This result allows us to pose the fractional Fourier transform as a tool for analyzing and describing optical systems composed of an arbitrary sequence of thin lenses and sections of free space and to arrive at a general class of fractional Fourier-transforming systems with variable input and output scale factors.

Key words: Diffraction, Fourier optics, optical information processing, fractional Fourier transforms.

\section{INTRODUCTION}

The $a$ th-order fractional Fourier transform $\left(\mathcal{F}^{a} \hat{q}\right)(u)$ of the function $\hat{q}(u)$ is defined for $0<|a|<2$ as

$$
\begin{aligned}
&\left(\mathcal{F}^{a} \hat{q}\right)(u) \equiv \int_{-\infty}^{\infty} B_{a}\left(u, u^{\prime}\right) \hat{q}\left(u^{\prime}\right) \mathrm{d} u^{\prime}, \\
& B_{a}\left(u, u^{\prime}\right) \equiv \frac{\exp [-i(\pi \hat{\phi} / 4-\phi / 2)]}{|\sin \phi|^{1 / 2}} \\
& \times \exp \left[i \pi\left(u^{2} \cot \phi-2 u u^{\prime} \csc \phi+u^{\prime 2} \cot \phi\right)\right],
\end{aligned}
$$

where

$$
\phi \equiv \frac{a \pi}{2}
$$

and $\hat{\phi}=\operatorname{sgn}(\sin \phi)$. The kernel is defined separately for $a=0$ and $a= \pm 2$ as $B_{0}\left(u, u^{\prime}\right) \equiv \delta\left(u-u^{\prime}\right)$ and $B_{ \pm 2}\left(u, u^{\prime}\right) \equiv \delta\left(u+u^{\prime}\right)$, respectively. ${ }^{1,2}$ One may easily extend the definition outside the interval $[-2,2]$ by noting that $\mathcal{F}^{4 j+a} \hat{q}=\mathcal{F}^{a} \hat{q}$ for any integer $j$. Both $u$ and $u^{\prime}$ are interpreted as dimensionless variables.

Some essential properties of the fractional Fourier transform are as follows: (i) It is linear. (ii) The first-order transform $(a=1)$ corresponds to the common Fourier transform. (iii) It is additive in index, $\mathcal{F}^{a_{1}} \mathcal{F}^{a_{2}} \hat{q}=\mathcal{F}^{a_{1}+a_{2}} \hat{q}$. Other properties may be found in Refs. 1-11.

Optical implementations of the fractional Fourier transform have already been presented. In Refs. 3-7 we discussed the fractional Fourier-transforming property of quadratic graded-index media. Lohmann suggested two systems consisting of thin lenses separated by free space. ${ }^{9}$ The fact that the two approaches were equivalent and represented the fractional Fourier transform as defined in Refs. 1 and 2 was demonstrated in Ref. 10. Applications have been suggested in these references and in Refs. 7, 11, and 12. Later research ${ }^{13,14}$ provided certain extensions and experimental verification of the above results.
In this paper we derive a fundamental result stating that there exists a fractional Fourier-transform relation between the (appropriately scaled) optical amplitude distributions on two spherical reference surfaces with given radii and separation (Fig. 1 below). This result provides an alternative statement of the law of propagation and allows us to pose the fractional Fourier transform as a tool for analyzing and describing a rather general class of optical system. (Previous research has been primarily concerned with offering optical realization of the fractional Fourier transform. These realizations follow as special cases or applications of our more general formulation, which allows us to state the necessary and sufficient conditions for a fractional Fourier transform in full generality.)

After discussing in some detail the above-mentioned result, we show how the Fresnel diffraction integral can be expressed in terms of a fractional Fourier transform. We discuss how axially centered systems composed of an arbitrary number of lenses separated by arbitrary distances can be analyzed by means of fractional Fourier transforms. As an instructive example, we concentrate on the classical single-lens imaging configuration. We also specify the general conditions under which an arbitrary system is a fractional Fourier transformer.

Whenever we can express the result of an optical problem (such as Fraunhofer diffraction) in terms of a Fourier transform, we tend to think of this result as simple and elegant. This is justified by the fact that the Fourier transform has many simple and useful properties that make working with it attractive. The Fourier transform and image occur at certain privileged planes in an optical system. Often all our intuition about what happens in between these planes is that the amplitude distribution is given by a complicated integral. In this paper we show that the distribution of light at intermediate planes can be expressed in terms of the fractional Fourier transform (which also has several useful properties and operational formulas). Thus the fractional Fourier transform 
completes in a natural way the study of optical systems often called Fourier optics.

We note in passing that, although one-dimensional signals are considered throughout this paper for notational simplicity, straightforward generalization of the results to two dimensions is possible. Although for onedimensional systems it is more correct to speak of circular (or cylindrical) surfaces, here we customarily speak of spherical surfaces.

As a final word on terminology, we believe that ultimately the term Fourier transform should mean, in general, fractional Fourier transform and that the currently standard Fourier transform should be referred to as the first-order Fourier transform. Likewise, DFT should denote the discrete (fractional) Fourier transform, etc., and the invention of new acronyms and abbreviations should be discouraged. Then, instead of speaking of fractional Fourier optics, we will be able to speak simply of Fourier optics.

\section{FRACTIONAL FOURIER- TRANSFORMING PROPERTY OF PROPAGATION THROUGH FREE SPACE}

We refer to Fig. 1. The complex amplitude distributions with respect to the first and the second spherical reference surfaces are denoted by $q_{1}\left(x^{\prime}\right)$ and $q_{2}(x)$, respectively. The distributions with respect to the planar surfaces tangent to the spherical surfaces on the optical axis are likewise denoted by $p_{1}\left(x^{\prime}\right)$ and $p_{2}(x)$. If the radii of the spherical surfaces are denoted by $R_{1}$ and $R_{2}$, we have

$$
\begin{aligned}
p_{2}(x) & =q_{2}(x) \exp \left(i \pi x^{2} / \lambda R_{2}\right), \\
p_{1}\left(x^{\prime}\right) & =q_{1}\left(x^{\prime}\right) \exp \left(i \pi x^{\prime 2} / \lambda R_{1}\right),
\end{aligned}
$$

where $\lambda$ is the wavelength. Assuming propagation from left to right, $p_{2}(x)$ is related to $p_{1}\left(x^{\prime}\right)$ by a Fresnel integral:

$$
p_{2}(x)=\frac{\exp (i 2 \pi d / \lambda)}{\sqrt{i \lambda d}} \int_{-\infty}^{\infty} \exp \left[i \pi\left(x-x^{\prime}\right)^{2} / \lambda d\right] p_{1}\left(x^{\prime}\right) \mathrm{d} x^{\prime} .
$$

Combining Eqs. (3)-(5), we can obtain a relation between $q_{1}\left(x^{\prime}\right)$ and $q_{2}(x)$. To permit comparison of this relation with Eq. (1) we introduce the dimensionless variables $u^{\prime} \equiv$ $x^{\prime} / s_{1}$ and $u \equiv x / s_{2}$, where $s_{1}$ and $s_{2}$ are real-valued scale parameters with dimensions of length. Also introducing the hatted functions $\hat{q}_{1}\left(u^{\prime}\right) \equiv q_{1}\left(u^{\prime} s_{1}\right)$ and $\hat{q}_{2}(u) \equiv q_{2}\left(u s_{2}\right)$, we obtain

$$
\begin{aligned}
\hat{q}_{2}(u)= & \frac{\exp (i 2 \pi d / \lambda) s_{1}}{\sqrt{i \lambda d}} \\
& \times \int_{-\infty}^{\infty} \exp \left[\frac{i \pi}{\lambda d}\left(g_{2} s_{2}{ }^{2} u^{2}-2 s_{1} s_{2} u u^{\prime}+g_{1} s_{1}{ }^{2} u^{\prime 2}\right)\right] \\
& \times \hat{q}_{1}\left(u^{\prime}\right) \mathrm{d} u^{\prime}
\end{aligned}
$$

where we established the definitions

$$
\begin{aligned}
& g_{1} \equiv 1+d / R_{1}, \\
& g_{2} \equiv 1-d / R_{2} .
\end{aligned}
$$

Now, comparing this result with the definition of the fractional Fourier transform [Eq. (1)], we conclude that $\hat{q}_{2}(u)$ is proportional to the fractional Fourier transform of $\hat{q}_{1}\left(u^{\prime}\right)$, i.e.,

$$
\begin{aligned}
\hat{q}_{2}(u)= & \left\{\frac{\exp [i 2 \pi d / \lambda) s_{1} \exp [i(\pi \hat{\phi} / 4-\phi / 2)]|\sin \phi|^{1 / 2}}{\sqrt{i \lambda d}}\right\} \\
& \times\left(\mathcal{F}^{a} \hat{q}_{1}\right)(u),
\end{aligned}
$$

if and only if

$$
\begin{aligned}
g_{2} \frac{s_{2}^{2}}{\lambda d} & =\cot \phi, \\
g_{1} \frac{s_{1}^{2}}{\lambda d} & =\cot \phi, \\
\frac{s_{1} s_{2}}{\lambda d} & =\csc \phi .
\end{aligned}
$$

These three equations are the necessary and sufficient conditions for Eq. (9) to hold.

We now discuss the consequences of these equations from three perspectives.

\section{A. Analysis}

1. Problem

Given $R_{1}, R_{2}$, and $d$, find $s_{1}, s_{2}$, and $a$ (or, equivalently, $\phi \equiv a \pi / 2)$. That is, we are given the reference surfaces and wish to find the order and the scale parameters of the resulting transform.

Equations (10) and (11) imply that

$$
g_{1} s_{1}^{2}=g_{2} s_{2}^{2} .
$$

Now, using the identity $\cot ^{2} \phi+1=\csc ^{2} \phi$ and Eqs. (10)-(13), we obtain

$$
\begin{aligned}
& s_{2}{ }^{4}=(\lambda d)^{2}\left(g_{2} / g_{1}-g_{2}{ }^{2}\right)^{-1}, \\
& s_{1}{ }^{4}=(\lambda d)^{2}\left(g_{1} / g_{2}-g_{1}{ }^{2}\right)^{-1} .
\end{aligned}
$$

Note that Eq. (13) implies that $g_{1} g_{2} \geq 0$ and that Eqs. (14) and (15) imply that $\left(g_{2} / g_{1}-g_{2}^{2}\right) \geq 0$ and $\left(g_{1} / g_{2}-g_{1}^{2}\right) \geq 0$. These conditions can be summarized in the form

$$
0 \leq g_{1} g_{2} \leq 1 .
$$

If a fractional transform relation is to hold, $R_{1}, R_{2}$, and $d$ must be specified such that this condition holds. (It

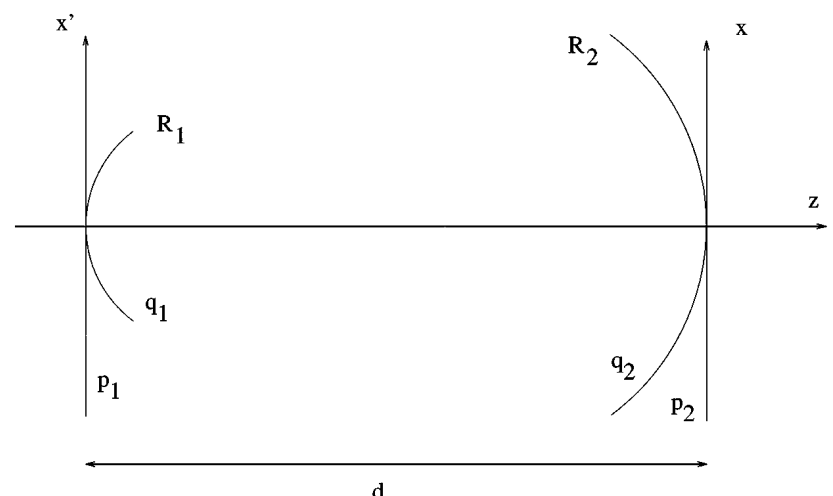

Fig. 1. Two spherical surfaces. This figure is drawn such that $R_{1}<0$ and $R_{2}>0$. The distance $d$ is always taken to be positive. 
is interesting that if Fig. 1 is interpreted as a spherical mirror resonator, this equation is the stability or the confinement condition of the resonator. ${ }^{15}$ )

We can see that, given $R_{1}, R_{2}$, and $d,\left|s_{2}\right|$ and $\left|s_{1}\right|$ are uniquely determined by Eqs. (14) and (15). Then Eq. (10) or (11) enables us to determine $\phi$ according to

$$
\tan \phi= \pm\left[\left(1 / g_{1} g_{2}\right)-1\right]^{1 / 2}
$$

where the \pm is determined according to the common sign of $g_{1}$ and $g_{2}$. The ambiguity in the inverse tangent function is resolved by examination of the sign of $\csc \phi$. Equation (12) tells us that, if we choose the signs of $s_{1}$ and $s_{2}$ such that $s_{1} s_{2} \geq 0$, then $\csc \phi \geq 0$, so that $\phi$ lies in $[0, \pi]$. In contrast, if $s_{1} s_{2} \leq 0$, then $\csc \phi \leq 0$, so that $\phi$ lies in $[-\pi, 0]$. These results are consistent with inversion and parity properties of the fractional Fourier transform.

\section{Result}

A fractional Fourier-transform relation exists between two spherical surfaces of radii $R_{1}, R_{2}$ and separation $d$ if and only if $0 \leq g_{1} g_{2} \leq 1$. If this condition is satisfied, $\left|s_{1}\right|$ and $\left|s_{2}\right|$ are determined by Eqs. (14) and (15), and $\phi$ is determined within $\pm \pi$ by Eq. (17). The quadrant of $\phi$ is determined by our choice of the signs of $s_{1}$ and $s_{2}$, or vice versa.

\section{B. Synthesis}

\section{Problem}

Given $s_{1}, s_{2}$, and $a$ (or, equivalently, $\phi \equiv a \pi / 2$ ), find $R_{1}, R_{2}$, and $d$. That is, we wish to design a fractional Fourier-transform system with specific order and scale factors.

Notice that Eq. (12) implies that the sign of $s_{1} s_{2}$ must be the same as the sign of $\csc \phi$, which is the same as the sign of $\phi$. If $s_{1}, s_{2}$, and $\phi$ have been specified consistent with this requirement, Eq. (12) determines $d$ :

$$
d=\left(s_{1} s_{2} / \lambda\right) \sin \phi
$$

Then Eqs. (10) and (11) give $g_{1}$ and $g_{2}$, which in turn give $R_{1}$ and $R_{2}$ :

$$
\begin{aligned}
& 1+d / R_{1} \equiv g_{1}=\left(s_{2} / s_{1}\right) \cos \phi, \\
& 1-d / R_{2} \equiv g_{2}=\left(s_{1} / s_{2}\right) \cos \phi .
\end{aligned}
$$

Note that $g_{1}$ and $g_{2}$, as given by these equations, will always satisfy $g_{1} g_{2}=\cos ^{2} \phi$, and thus $0 \leq g_{1} g_{2} \leq 1$.

\section{Result}

A fractional Fourier-transform relation of order $a$ between two spherical surfaces with the input and the output scaled by $s_{1}$ and $s_{2}$, respectively, can be obtained if and only if $\operatorname{sgn}\left(s_{1} s_{2}\right)=\operatorname{sgn}(\sin \phi)$. If this condition is satisfied, we must choose $R_{1}, R_{2}$, and $d$ according to Eqs. (18)-(20).

\section{Propagation}

\section{Problem}

Given $s_{1}, R_{1}$, and $d$, find $a$ (or $\phi$ ), $s_{2}$, and $R_{2}$. That is, given the radius of the spherical reference surface and the scale parameter on the input side, find them at a distance $d$ to the right, as well as the order of the resulting transform at that distance.

Because $R_{1}$ and $d$ are given, $g_{1}$ is also known. Using Eqs. (10)-(12), we can obtain

$$
\begin{aligned}
\tan \phi & =\frac{\lambda d}{g_{1} s_{1}{ }^{2}}, \\
s_{2}{ }^{2} & =g_{1}{ }^{2} s_{1}{ }^{2}+\frac{(\lambda d)^{2}}{s_{1}{ }^{2}}, \\
1-d / R_{2} & \equiv g_{2}=\frac{g_{1} s_{1}{ }^{4}}{g_{1}{ }^{2} s_{1}{ }^{4}+(\lambda d)^{2}} .
\end{aligned}
$$

We are free to choose the sign of $s_{2}$, which together with the specified sign of $s_{1}$ determines the sign of $\csc \phi$. This sign determines the quadrant of $\phi$ in Eq. (21). If we assume that $s_{1}, s_{2}$ are both positive, $\phi$ lies in the interval $[0, \pi]$. Now $\phi$, as given by Eq. (21), is a continuous monotonic increasing function of $d$. Let us assume that the location of the first surface is fixed at the origin $z=0$ of the optical axis and that we examine the distribution of light after it has propagated a distance $z=d$ in the positive $z$ direction. For larger values of $d$, we observe fractional transforms of the initial distribution of larger-order $\phi$; the amplitude distribution of light is continuously fractional Fourier transformed as it propagates.

2. Result

Given the distribution of light on a spherical reference surface of radius $R_{1}$ and scale parameter $s_{1}$, we can observe its fractional Fourier transform on another spherical reference surface a distance $d$ to the right. The radius $R_{2}$ and scale parameter $s_{2}$ for this surface and the order of the fractional transform are given by Eqs. (21)-(23), where the quadrant of $\phi$ is determined by our choice of the sign of $s_{2}$.

\section{ILLUSTRATIVE APPLICATIONS}

We now consider some applications of the above results. For convenience we restrict $s_{1}$ and $s_{2}$ to positive values. This implies $\csc \phi>0$, so that $\phi$ lies in the interval $[0, \pi]$. Using Eq. (12) we can now write Eq. (9) in the more meaningful form

$$
\hat{q}_{2}(u)=\left[\exp (i 2 \pi d / \lambda) \exp (-i a \pi / 4) \sqrt{s_{1} / s_{2}}\right] \quad\left(\mathcal{F}^{a} \hat{q}_{1}\right)(u) .
$$

The phase factor $\exp (i 2 \pi d / \lambda)$ is associated with propagation over the distance $d$. The phase factor $\exp (-i \phi / 2)$ is the Gouy phase shift. ${ }^{15}$ The factor $\sqrt{s_{1} / s_{2}}$ ensures power conservation.

\section{A. Fresnel Diffraction as a Fractional Fourier Transform}

Let us assume that a plane wave of unit amplitude illuminates a planar screen with complex amplitude transmittance $t(x)$. We can handle this case by letting $R_{1} \rightarrow \infty$, as given in Fig. 1. We then have $q_{1}\left(x^{\prime}\right)=p_{1}\left(x^{\prime}\right)=t\left(x^{\prime}\right)$. Because $g_{1}=1$ in this case, Eq. (11) implies that $\cot \phi \geq$ 0 , so that $\phi$ lies in the interval $[0, \pi / 2]$. 
We also assume that the scale parameter $s_{1}$ associated with the planar reference plane is specified freely. Then Eq. (11) gives us the order of the fractional transform observed at a distance $d$ from the screen. The scale $s_{2}$ of the transform at this distance can then be found from Eq. (12). Then Eq. (10) yields $g_{2}$ and hence $R_{2}$. Thus the observed field $p_{2}(x)$ at a distance $d>0$ is given by

$$
\begin{aligned}
p_{2}(x)= & {\left[\exp (i 2 \pi d / \lambda) \exp (-i a \pi / 4) \sqrt{s_{1} / s_{2}}\right] \exp \left[\left(i \pi x^{2} / \lambda R_{2}\right)\right] } \\
& \times\left(\mathcal{F}^{a} \hat{t}\right)\left(x / s_{2}\right),
\end{aligned}
$$

with

$$
\begin{aligned}
\frac{a \pi}{2} & \equiv \phi=\arctan \left(\frac{\lambda d}{s_{1}^{2}}\right) \\
s_{2} & =s_{1}\left[1+\frac{(\lambda d)^{2}}{s_{1}^{4}}\right]^{1 / 2}, \\
R_{2} & =d\left[1+\frac{s_{1}^{4}}{(\lambda d)^{2}}\right],
\end{aligned}
$$

where $\hat{t}\left(u^{\prime}\right) \equiv t\left(u^{\prime} s_{1}\right)$. We can eliminate the spherical phase factor $\exp \left(i \pi x^{2} / \lambda R_{2}\right)$ by choosing a spherical reference surface with radius $R_{2}$, i.e., if we observe $g_{2}(x)$ instead of $p_{2}(x)$. (The various phase factors would have no effect if we were observing only the intensity.)

Thus we conclude that the Fresnel diffraction integral can be formulated as a fractional Fourier transform. As $d$ is increased from 0 to $\infty$, the order $a$ of the fractional transform increases according to Eq. (26) from 0 to 1. Letting $d \rightarrow \infty$, we obtain the intuitively appealing $a=1$, $s_{2}=\lambda d / s_{1}$, and $R_{2}=d$, which we readily associate with the Fraunhofer diffraction pattern, which is nothing but the Fourier transform of the diffracting screen.

\section{B. Symmetric Case}

Referring to Fig. 1, let us consider the special case in which $-R_{1}=R_{2} \equiv R$ and $g_{1}=g_{2} \equiv g \equiv 1-d / R$. Equations (10) and (11) then imply that $s_{1}=s_{2}$, which we denote by $s$. The condition $0 \leq g_{1} g_{2} \leq 1$ becomes $0 \leq g^{2} \leq 1$ or, more simply, $|g| \leq 1$. This result implies that $0 \leq d / R \leq 2$ and hence that $R \geq d / 2$.

Equations (10) and (12) now become

$$
\begin{aligned}
g\left(s^{2} / \lambda d\right) & =\cot \phi, \\
s^{2} / \lambda d & =\csc \phi,
\end{aligned}
$$

from which it also follows that

$$
g=\cos \phi .
$$

Given $R$ and $d$ such that $|g| \leq 1$, Eq. (31) immediately determines $\phi$. Then either Eq. (29) or (30) yields $s$. Alternatively, given $\phi$ and $s$, we can use the same equations to find $d$ and $g$ and hence $R$.

\section{Fractional Fourier Transform between Planar Surfaces}

We have seen that there exists a fractional Fouriertransform relation between two spherical surfaces, as depicted in Fig. 1. By using a lens to compensate the spherical phase factors at both surfaces, we can obtain a fractional Fourier transform between two planar surfaces.
We simply choose lenses with focal lengths $f_{1}=-R_{1}$ and $f_{2}=R_{2}$. Thus, with our synthesis result, it is possible to design a fractional Fourier transformer of given order $a$ and of desired input and output scale parameters $s_{1}$ and $s_{2}$.

Let us restrict ourselves to the symmetric case for which $R \geq d / 2>0$. Then, using two positive lenses of focal length $f=R>0$, we obtain Lohmann's Type II fractional Fourier-transforming system (Fig. 2). ${ }^{9}$ If the order of the fractional transform $a \pi / 2 \equiv \phi$ and the scale $s$ are specified, then the separation of the lenses $d$ and their focal length $f$ must be chosen as

$$
\begin{aligned}
& d=\left(s^{2} / \lambda\right) \sin \phi, \\
& f=\left(s^{2} / \lambda\right) \cot (\phi / 2) .
\end{aligned}
$$

Alternatively, let us consider an asymmetric pair of spherical surfaces with $R_{1} \rightarrow \infty$ and $R_{2}=R$. Let us place immediately to the right of the second surface a thin lens of focal length $f=R / 2$, whose effect will be to map the amplitude distribution on the spherical surface of radius $R$ onto a spherical surface of radius $-R$. Now let us place after the lens a second asymmetric pair of spherical surfaces with $R_{1}=-R$ and $R_{2} \rightarrow \infty$. The overall system consists of a stretch of free space followed by a lens followed by another stretch of free space, which is nothing but Lohmann's Type I fractional Fourier-transforming system (Fig. 3). Its analysis is similar to what has been presented above and is thus not repeated.

We refer to such realizations of the fractional Fourier transform as canonical realizations Type II and Type I.

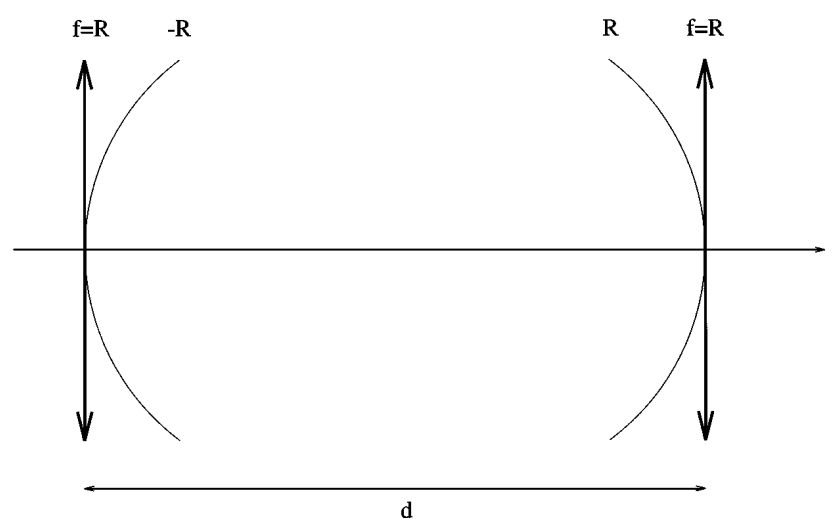

Fig. 2. Canonical realization Type II.

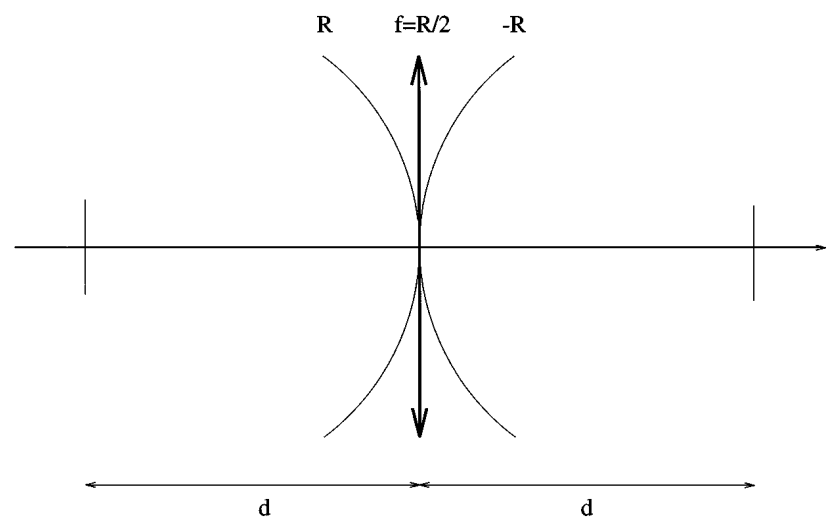

Fig. 3. Canonical realization Type I. 
They are summarized below within the framework of this paper. An alternative discussion of these systems is to be found in Lohmann's paper. ${ }^{9}$

Canonical fractional Fourier-transforming configurations have planar input and output reference surfaces separated by a distance $l$ and with scale parameter $s$. The focal lengths of the lenses are denoted by $f$. The output $p_{\text {out }}$ of such a system is related to the input $p_{\text {in }}$ by the relation

$$
\hat{p}_{\text {out }}(u)=\exp (i 2 \pi l / \lambda) \exp (-i a \pi / 4)\left(\mathcal{F}^{a} \hat{p}_{\text {in }}\right)(u),
$$

where $u \equiv x / s_{2}, u^{\prime} \equiv x^{\prime} / s_{1}$ and $\hat{p}_{\text {out }}(u) \equiv p_{\text {out }}\left(u s_{2}\right)$, $\hat{p}_{\text {in }}\left(u^{\prime}\right) \equiv p_{\text {in }}\left(u^{\prime} s_{1}\right)$. We can see that the output is essentially the ath-order fractional Fourier transform of the input.

1. Type $I I$

A Type II system consists of a lens of focal length $f$ followed by a section of free space of length $d$ followed by a second lens of focal length $f$ (Fig. 2). Thus its total length is $l=d$. We can obtain $d$ and $f$ in terms of $s$ and $\phi$ (or $a$ ) by using

$$
\begin{aligned}
& d=\left(s^{2} / \lambda\right) \sin \phi, \\
& f=\left(s^{2} / \lambda\right) \cot (\phi / 2) .
\end{aligned}
$$

Alternatively, provided that $f \geq d / 2$, we can find $\phi$ (or $a$ ) and $s$ in terms of $d$ and $f$ by using

$$
\begin{aligned}
\phi & =\arccos (1-d / f), \\
s^{4} & =\frac{\lambda^{2} d f}{2-d / f} .
\end{aligned}
$$

\section{Tyре $I$}

A Type I system consists of a section of free space of length $d$ followed by a lens of focal length $f$ followed by a second section of free space of length $d$ (Fig. 3). Thus its total length is $l=2 d$. We can obtain $d$ and $f$ in terms of $s$ and $\phi$ (or $a$ ) by using

$$
\begin{aligned}
& d=\left(s^{2} / \lambda\right) \tan (\phi / 2), \\
& f=\left(s^{2} / \lambda\right) \csc \phi .
\end{aligned}
$$

Alternatively, we can find $\phi$ (or $a$ ) and $s$ in terms of $d$ and $f$ by using

$$
\begin{aligned}
\phi & =\arccos (1-d / f), \\
s^{4} & =\lambda^{2} d f(2-d / f) .
\end{aligned}
$$

\section{Classical Single-Lens Imaging}

Consider the classical single-lens imaging configuration in which the object is located a distance $d_{0}>0$ to the left of the lens and the image is located a distance $d_{i}>0$ to the right of the lens, which has focal length $f>0$ (Fig. 4). We can view this system as performing two consecutive fractional Fourier-transform operations (from the object to the lens and from the lens to the image), provided that the radius $R_{-}$of the spherical reference surface just before the lens and the radius $R_{+}$of the spherical reference surface just after the lens are related by

$$
1 / R_{+}=\left(1 / R_{-}\right)-(1 / f)
$$

This equation states that the amplitude distribution of light on the reference surface with radius $R_{-}$is mapped by the lens onto a reference surface with radius $R_{+}$. If we are to look into the combined effect of the two consecutive fractional transforms, we should choose the scale factors $s_{-}$and $s_{+}$immediately before and immediately after the lens such that

$$
s_{+}=s_{-} .
$$

Letting $\phi_{o} \equiv a_{o} \pi / 2$ and $\phi_{i} \equiv a_{i} \pi / 2$ denote the order of transformation occurring from the object to the lens and from the lens to the image, respectively, we can write the imaging condition (for an inverted image) as $a_{o}+a_{i}= \pm 2$ or, equivalently,

$$
\phi_{o}+\phi_{i}= \pm \pi
$$

This condition follows from the fact that $\mathcal{F}^{ \pm 2}[\hat{q}(u)]=$ $\hat{q}(-u)$ for any $\hat{q}(u)$.

Now, with the definitions $g_{-} \equiv 1-d_{o} / R_{-}, g_{+} \equiv 1+$ $d_{i} / R_{+}$, we have [see Eqs. (10) and (11)]

$$
\begin{aligned}
& \cot \phi_{o}=g_{-}\left(s_{-}{ }^{2} / \lambda d_{o}\right), \\
& \cot \phi_{i}=g_{+}\left(s_{+}{ }^{2} / \lambda d_{i}\right) .
\end{aligned}
$$

It is now possible to show that Eq. (45) implies the wellknown imaging condition

$$
1 / f=\left(1 / d_{o}\right)+\left(1 / d_{i}\right)
$$

Furthermore, if we let $s_{o}$ and $s_{i}$ denote the scale factors associated with the object and the image, Eq. (12) lets us write

$$
\begin{aligned}
& s_{o} s_{-} / \lambda d_{o}=\csc \phi_{o}, \\
& s_{+} s_{i} / \lambda d_{i}=\csc \phi_{i} .
\end{aligned}
$$

Now, because Eq. (45) implies that $\csc \phi_{o}=\csc \phi_{i}$, we obtain the magnification $M$ of the scale factors

$$
M \equiv s_{i} / s_{o}=d_{i} / d_{o},
$$

again a familiar result.

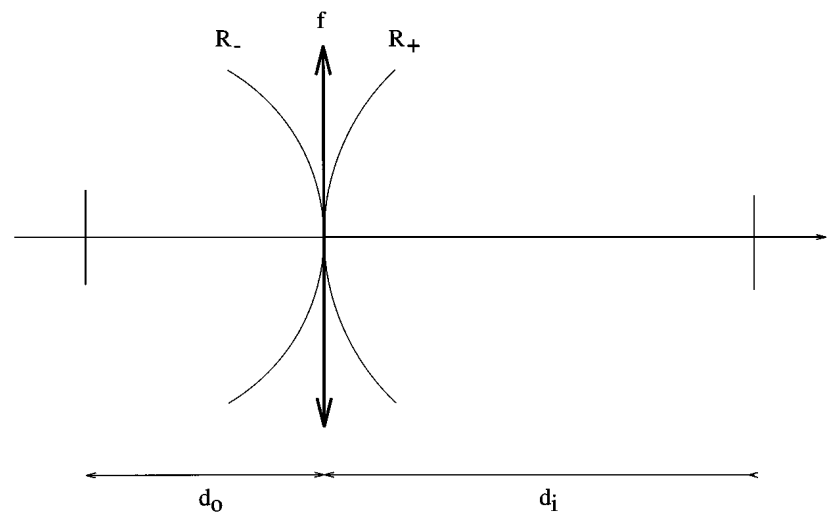

Fig. 4. Single-lens imaging. 


\section{E. General Lens Systems Analyzed as Consecutive Fractional Fourier Transforms}

More-complicated systems involving several lenses separated by arbitrary distances can be analyzed in a similar manner. For simplicity let us restrict ourselves to positive lenses. Let the distance separating the input (or the object) from the first lens be $d_{0}$; the distance separating the first and the second lens, $d_{1}$; and the distance separating the $i$ th and the $(i+1)$ th lens, $d_{i}$. The focal length of the $i$ th lens is denoted by $f_{i}$. In general, the subscripts $i-$ and $i+$ are used to denote quantities immediately to the left and the right of the $i$ th lens, respectively. $\phi_{i}$ denotes the order of the fractional transform associated with propagation from lens $i$ to lens $i+1$.

Assume that the input is specified with respect to a particular spherical reference surface of radius $R_{0+}$ and that the input scale parameter is denoted by $s_{0+}$. Just before the first lens in the system we observe the fractional Fourier transform of the input of order $\phi_{0}$, where $\phi_{0}$ can be calculated from cot $\phi_{0}=g_{0+} s_{0+}{ }^{2} / \lambda d_{0}$ [Eq. (11)], with $g_{0+} \equiv 1+d_{0} / R_{0+}$. The scale of this transform is $s_{1-}=\left(\lambda d_{0} / s_{0+}\right) \csc \phi_{0}$ [Eq. (12)], and the transform is observed on a spherical reference surface of radius $R_{1-}$, which we can calculate from $g_{1-} \equiv 1-d_{0} / R_{1-}$ and $g_{1-}=$ $\left(\lambda d_{0} / s_{1-}{ }^{2}\right) \cot \phi_{0}$ [Eq. (10)].

Now we can make our way through the lens, using $s_{1+}=s_{1-}$ and $1 / R_{1+}=1 / R_{1-}-1 / f_{1}$ [Eqs. (43) and (44)]. Then, just before the second lens, on a spherical reference surface of radius $R_{2-}$, we observe the fractional Fourier transform of the input of order $\phi_{0}+\phi_{1}$, and so on.

Let us assume that all $d_{0}, d_{1}, \ldots, f_{1}, f_{2}, \ldots$, as well as $s_{0+}$ and $R_{0+}$, are specified. Then we can work our way through the system iteratively, using the following equations for $i=0,1,2, \ldots$ :

$$
\begin{aligned}
g_{i+} & =1+d_{i} / R_{i+}, \\
\cot \phi_{i} & =g_{i+} s_{i+}{ }^{2} / \lambda d_{i}, \\
s_{(i+1)-} & =\left(\lambda d_{i} / s_{i+}\right) \csc \phi_{i}, \\
s_{(i+1)+} & =s_{(i+1)-}, \\
g_{(i+1)-} & =\left[\lambda d_{i} / s_{(i+1)-}{ }^{2}\right] \cot \phi_{i}, \\
R_{(i+1)-} & =\frac{d_{i}}{1-g_{(i+1)-}}, \\
R_{(i+1)+} & =\frac{f_{i+1} R_{(i+1)-}}{f_{i+1}-R_{(i+1)-}} .
\end{aligned}
$$

Here $s_{i+}$ and $R_{i+}$ may be considered to be the state variables of the iteration. The cumulative order of the transform just before the $i$ th lens is $\phi_{\mathrm{cum}_{i}}=\phi_{0}+\phi_{1}+\cdots+$ $\phi_{i-1}$. This procedure allows us to find the orders of the transforms at the lenses, but of course it is also possible to calculate the order of the transform observed at any intermediate location (the propagation result).

Thus we can see that, in an optical system involving many lenses separated by arbitrary distances, the amplitude distribution is continuously fractional Fourier transformed as it propagates through the system. The order $\phi(z)$ of the fractional transform observed at the distance $z$ along the optical axis is a monotonically increasing function. The transforms are observed on spherical ref- erence surfaces. Wherever the order of the transform $\phi(z)$ is equal to $(4 j+1) \pi / 2$ for any integer $j$, we observe the Fourier transform of the input. Wherever the order is equal to $(4 j+2) \pi / 2$, we observe an inverted image, etc.

The results of this subsection can be generalized for systems composed of an arbitrary sequence of spherical refracting surfaces.

F. Interpretation of Quadratic Graded-Index Media as a Continuum of Infinitesimal Thin Lenses Interspersed with Infinitesimal Sections of Free Space

The fractional Fourier-transforming property of quadratic graded-index media was discussed in Refs. 4-7 and 10. The refractive index in such a medium has the following dependence on $x$ :

$$
n^{2}(x)=n_{0}^{2}\left[1-(x / \xi)^{2}\right],
$$

where $n_{0}>0$ and $\xi>0$ are the medium parameters. In considering such a medium of length $l$, the output is related to the input by Eq. (34) just as well, provided that we replace $\lambda \rightarrow \lambda / n_{0}$. Now $\phi \equiv a \pi / 2$ and $s>0$ are given in terms of $n_{0}, \xi$, and $l$ by

$$
\begin{aligned}
\phi & =l / \xi, \\
s^{2} & =\lambda \xi / n_{0} .
\end{aligned}
$$

Now we discuss the relation between such fractional Fourier transformers and the canonical systems discussed in Subsection 3.C. Let us consider Type I (the discussion is similar for Type II). Assume that we cascade $N$ Type I systems, each of length $2 d$, so that the overall length of the system is $l=N 2 d$. Now, keeping this overall length and the scale parameter $s$ fixed, we let $N \rightarrow \infty$ and $2 d \rightarrow 0$. Physically, what we obtain is a large number of closely spaced weak-focal-power lenses. ${ }^{16}$ In the limit the average refractive-index distribution of this assembly will have a quadratic dependence on $x$, similar to Eq. (59).

To see that this is true, let us consider optical paths parallel to the optical axis. Using small $\phi$ approximations of Eqs. (39) and (40), we can show that the phase collected along such a path through the infinite cascade of infinitesimal Type I systems is

$$
\simeq-\pi \phi(x / s)^{2},
$$

where we have dropped the constant term $2 \pi N 2 d / \lambda$. Turning our attention to quadratic index media, Eqs. (59)-(61) allow us to write the collected phase in exactly the same form as above. This means that if we assume functional equivalence of the two systems (identical values of $\phi$ and $s$ ) we can deduce their physical equivalence (identical collected phase, which is proportional to optical density).

From the same sets of equations we can derive another relation that is valid for both cases:

$$
\phi=\left(\lambda / s^{2}\right) \ell,
$$

where $\lambda \rightarrow \lambda / n_{0}$ for the quadratic index case. 


\section{GENERAL CONDITIONS FOR THE OPTICAL FRACTIONAL FOURIER TRANSFORM}

The class of quadratic-phase systems ${ }^{17-21}$ is characterized by linear transformations of the form

$$
\begin{aligned}
p_{\text {out }}(x) & =\int_{-\infty}^{\infty} h\left(x, x^{\prime}\right) p_{\text {in }}\left(x^{\prime}\right) \mathrm{d} x^{\prime}, \\
h\left(x, x^{\prime}\right) & =C \exp \left[i \pi\left(\alpha x^{2}-2 \beta x x^{\prime}+\gamma x^{\prime 2}\right)\right],
\end{aligned}
$$

where $C$ is a complex constant, and $\alpha, \beta$, and $\gamma$ are real constants independent of $x$ and $x^{\prime}$. Optical systems involving an arbitrary sequence of thin lenses and sections of free space (in the Fresnel approximation) belong to this class. (We can easily show this by first proving that the kernel for a section of free space followed by a lens and a lens followed by a section of free space both assume the above form and by then noting that an arbitrary system can be composed of these units.)

For this class of systems it is sufficient to specify the parameters $C, \alpha, \beta$, and $\gamma$ to characterize the system completely. The condition for a fractional Fourier transform can be stated in terms of these parameters as follows. There should exist scale parameters $s_{1}, s_{2}$ such that

$$
\begin{aligned}
\alpha s_{2}{ }^{2} & =\cot \phi=\gamma s_{1}{ }^{2}, \\
\beta s_{1} s_{2} & =\csc \phi
\end{aligned}
$$

for some $\phi$. It can be shown that such scale parameters can be found if and only if $0 \leq \alpha \gamma \leq \beta^{2}$. If this condition is satisfied, the necessary scale parameters and the order of the resulting transform are given by

$$
\begin{aligned}
{s_{1}}^{4} & =\left(\beta^{2} \gamma / \alpha-\gamma^{2}\right)^{-1}, \\
s_{2}{ }^{4} & =\left(\beta^{2} \alpha / \gamma-\alpha^{2}\right)^{-1}, \\
\tan \phi & = \pm\left(\beta^{2} / \alpha \gamma-1\right)^{1 / 2},
\end{aligned}
$$

where the sign of $\tan \phi$ is the same as the identical signs of $\alpha$ and $\gamma$ and where $\phi$ lies in the interval $[-\pi, 0]$ or $[0, \pi]$ according to whether $\beta s_{1} s_{2} \leq 0$ or $\beta s_{1} s_{2} \geq 0$. For example, if $s_{1} s_{2} \geq 0, \alpha \geq 0$, and $\beta \geq 0, \phi$ lies in the interval $[0, \pi / 2]$.

If the condition $0 \leq \alpha \gamma \leq \beta^{2}$ is not satisfied, it is still possible to observe a fractional Fourier transform between spherical (rather than planar) reference surfaces at the input and the output. (That is, it is possible to take any system and make a fractional Fourier transformer out of it by appending lenses to the input and the output.) To show this, we note that the kernel $h^{\prime}\left(x, x^{\prime}\right)$ between the new spherical reference surfaces will be modified according to $\alpha^{\prime}=\alpha-1 / \lambda R_{2}, \beta^{\prime}=\beta$, and $\gamma^{\prime}=\gamma+1 / \lambda R_{1}$, where $R_{1}$ and $R_{2}$ are the radii of the new input and output reference surfaces. Now we can always ensure that $0 \leq \alpha^{\prime} \gamma^{\prime} \leq \beta^{\prime 2}$ by an appropriate choice of $R_{1}$ and $R_{2}$.

It is possible to arrive at the same results through a different formalism. Let us define the transformation matrix characterizing the optical system as

$$
\begin{aligned}
{\left[\begin{array}{ll}
A & B \\
C & D
\end{array}\right] } & \equiv\left[\begin{array}{cc}
\gamma / \beta & 1 / \beta \\
-\beta+\alpha \gamma / \beta & \alpha / \beta
\end{array}\right] \\
& =\left[\begin{array}{cc}
\alpha / \beta & -1 / \beta \\
\beta-\alpha \gamma / \beta & \gamma / \beta
\end{array}\right]^{-1},
\end{aligned}
$$

with determinant $A D-B C=1$. There are many reasons for defining such a matrix. First, if several systems, each characterized by such a matrix, are cascaded, one can find the matrix characterizing the overall system by multiplying the matrices of the several systems. Second, the effect of the optical system on the Wigner distribution ${ }^{17,18}$ of the input is easily expressed in terms of this matrix. Third, this matrix can be made the basis of a geometricaloptics description of a quadratic-phase system; it is essentially the well-known ray matrix. ${ }^{15}$ These issues are discussed extensively in the research of Bastiaans, ${ }^{17-21}$ so they are not considered further here. (Bastiaans actually deals with the inverse of the above matrix.)

Introducing the variables $u \equiv x / s_{2}$ and $u^{\prime} \equiv x^{\prime} / s_{1}$ in Eqs. (64), one can write the kernel as $\propto \exp \left[i \pi\left(\bar{\alpha} u^{2}-\right.\right.$ $\left.\left.2 \bar{\beta} u u^{\prime}+\bar{\gamma} u^{\prime 2}\right)\right]$, with $\bar{\alpha}=\alpha s_{2}{ }^{2}, \bar{\beta}=\beta s_{1} s_{2}$, and $\bar{\gamma}=\gamma s_{1}{ }^{2}$. It is now possible to show that the elements of the new transformation matrix associated with the dimensionless variables are related to the elements of the original transformation matrix given in Eq. (70):

$$
\begin{aligned}
{\left[\begin{array}{ll}
\bar{A} & \bar{B} \\
\bar{C} & \bar{D}
\end{array}\right] } & =\left[\begin{array}{cc}
\bar{\gamma} / \bar{\beta} & 1 / \bar{\beta} \\
-\bar{\beta}+\bar{\alpha} \bar{\gamma} / \bar{\beta} & \bar{\alpha} / \bar{\beta}
\end{array}\right] \\
& =\left[\begin{array}{cc}
A s_{1} / s_{2} & B / s_{1} s_{2} \\
C s_{1} s_{2} & D s_{2} / s_{1}
\end{array}\right] .
\end{aligned}
$$

The transformation matrix associated with the fractional Fourier transform of order $\phi$ is the rotation matrix $^{4,9,10}$

$$
\left[\begin{array}{cc}
\cos \phi & \sin \phi \\
-\sin \phi & \cos \phi
\end{array}\right]
$$

Thus the condition for a fractional Fourier transform is that there exist scale parameters $s_{1}$ and $s_{2}$ such that the matrices given in expressions (71) and (72) are equal for some $\phi$. The necessary and sufficient condition for such scale parameters to exist can be expressed as $0 \leq A D \leq 1$ (or, equivalently, $-1 \leq B C \leq 0$ ). If this condition is satisfied, the necessary scale parameters and the order of the resulting transform are given by

$$
\begin{aligned}
s_{1}{ }^{4} & =B^{2}\left(A / D-A^{2}\right)^{-1}, \\
s_{2}{ }^{4} & =B^{2}\left(D / A-D^{2}\right)^{-1}, \\
\cos \phi & = \pm(A D)^{1 / 2},
\end{aligned}
$$

where $\operatorname{sgn}(\cos \phi)=\operatorname{sgn}\left(A s_{1} / s_{2}\right)$ and $\phi$ lies in the interval $[-\pi, 0]$ or $[0, \pi]$ according to whether $B / s_{1} s_{2} \leq 0$ or $B / s_{1} s_{2} \geq 0$. For example, if $s_{1} s_{2} \geq 0, A \geq 0$, and $B \geq 0$, $\phi$ lies in the interval $[0, \pi / 2]$.

If the condition $0 \leq A D \leq 1$ is not satisfied, we can still make a fractional Fourier transformer out of this system by appending lenses at its input and output surfaces. (That is, for any system, it is possible to find spherical input and output reference surfaces between which a fractional Fourier-transform relation exists.) To show this, we multiply the transformation matrix from the left and the right by the transformation matrix of a thin lens ${ }^{15}$ :

$$
\left[\begin{array}{ll}
A^{\prime} & B^{\prime} \\
C^{\prime} & D^{\prime}
\end{array}\right]=\left[\begin{array}{cc}
1 & 0 \\
-K_{2} & 1
\end{array}\right]\left[\begin{array}{ll}
A & B \\
C & D
\end{array}\right]\left[\begin{array}{cc}
1 & 0 \\
-K_{1} & 1
\end{array}\right],
$$


where $K_{1}, K_{2}$ are measures of the powers of the lenses. Multiplying the matrices, we find that $A^{\prime}=A-K_{1} B$ and $D^{\prime}=D-K_{2} B$. It is clearly possible to satisfy the condition $0 \leq A^{\prime} D^{\prime} \leq 1$ by an appropriate choice of $K_{1}$ and $K_{2}$.

\section{CONCLUSIONS}

There exists a fractional Fourier-transform relation between two spherical reference surfaces of given radii and separation. It is possible to determine the order and the scale parameters associated with this fractional transform, given the radii and the separation of the surfaces. Alternatively, given the desired order and scale parameters, it is possible to determine the necessary radii and separation.

The propagation of light along the $+z$ direction can be viewed as a process of continual fractional Fourier transformation. As light propagates, its distribution evolves through fractional transforms of increasing orders. The order $\phi(z)$ of the fractional transform observed at $z$ is a continuous monotonic increasing function of $z$. (The fractional transform at $z$ is observed on a spherical reference surface intersecting the optical axis at that location.) One of the central results of diffraction theory is that the far-field diffraction pattern is the Fourier transform of the diffracting object. Thus we have shown that the field at a closer distance is the fractional Fourier transform of the diffracting object.

The effect of a thin lens on such a propagating waveform is merely to bend the spherical reference surface into another reference surface with a different radius. Thus we can continue tracking the evolution of the wave in terms of fractional Fourier transforms, starting from this new reference surface.

It has been shown by Onural ${ }^{22}$ that the propagation of light in the Fresnel approximation can be viewed as a wavelet transform. The relation between fractional Fourier transforms and wavelet transforms is discussed in Ref. 7, so that Onural's result can be tied to ours.

We have discussed rather carefully what we have termed canonical fractional Fourier-transforming systems, which are the simplest symmetric configurations yielding a fractional Fourier transform between planar reference surfaces. We also showed that quadratic graded-index media can be viewed as the limit of a larger and larger number of weaker and weaker lenses.

We derived the (well-known) single-lens imaging equations, starting from an imaging condition stated in terms of fractional Fourier transforms. Generalizing, we saw that fractional Fourier transforms provide a new way of analyzing optical systems involving several lenses separated by arbitrary distances. Such systems can be analyzed by means of geometrical optics, Fresnel integrals (spherical-wave expansions), plane-wave expansions, Hermite-Gaussian beam expansions, and, as we showed, fractional Fourier transforms. The various approaches prove useful in different situations and provide different viewpoints that complement each other. The fractional Fourier-transform approach is appealing in that it describes the continuous evolution of the wave as it propagates through the system.

Finally, we considered a rather general class of optical systems and stated the general conditions that a member of this class must satisfy for its output to be viewed as the fractional Fourier transform of its input. We also showed that one can make a fractional Fourier transformer out of any system by appending lenses of appropriate focal length at the input and the output faces.

It is also of interest to formulate our results in the framework and the conventions of Hermite-Gaussian beams. This approach is suitable for studying beam propagation and spherical mirror resonators. ${ }^{14}$

We have avoided discussing complex-order transforms so as to avoid dealing with complex-valued scale parameters $s_{1}$ and $s_{2}$. Such systems do not satisfy $0 \leq g_{1} g_{2} \leq$ 1. Thus, if Fig. 1 is interpreted as a spherical mirror resonator, we are dealing with an unstable resonator. ${ }^{14}$ This case might be worth investigating.

\section{ACKNOWLEDGMENT}

It is a pleasure to acknowledge the contributions of Adolf W. Lohmann of the University of Erlangen-Nürnberg, which were made in the form of many discussions and suggestions. He has been a constant source of inspiration throughout this research.

Note added in proof: The reader is also referred to Refs. 23 and 24, two recent publications that discuss similar results.

\section{REFERENCES AND NOTES}

1. V. Namias, "The fractional Fourier transform and its application in quantum mechanics," J. Inst. Math. Its Appl. 25, 241-265 (1980).

2. A. C. McBride and F. H. Kerr, "On Namias's fractional Fourier transform," IMA J. Appl. Math. 39, 159-175 (1987)

3. D. Mendlovic, H. M. Ozaktas, and A. W. Lohmann, "Fourier transforms of fractional order and their optical interpretation," in Optical Computing, Vol. 7 of 1993 OSA Technical Digest Series (Optical Society of America, Washington, D.C., 1993), pp. 127-130.

4. H. M. Ozaktas and D. Mendlovic, "Fourier transforms of fractional order and their optical interpretation," Opt. Commun. 101, 163-169 (1993).

5. D. Mendlovic and H. M. Ozaktas, "Fractional Fourier transformations and their optical implementation. I," J. Opt. Soc. Am. A 10, 1875-1881 (1993).

6. H. M. Ozaktas and D. Mendlovic, "Fractional Fourier transforms and their optical implementation. II," J. Opt. Soc. Am. A 10, 2522-2531 (1993).

7. H. M. Ozaktas, B. Barshan, D. Mendlovic, and L. Onural, "Convolution, filtering, and multiplexing in fractional Fourier domains and their relation to chirp and wavelet transforms," J. Opt. Soc. Am. A 11, 547-559 (1994).

8. L. B. Almeida, "The fractional Fourier transform and timefrequency representations," IEEE Trans. Signal Process. 42, 3084 (1994).

9. A. W. Lohmann, "Image rotation, Wigner rotation, and the fractional Fourier transform," J. Opt. Soc. Am. A 10, 2181-2186 (1993)

10. D. Mendlovic, H. M. Ozaktas, and A. W. Lohmann, "Gradedindex fibers, Wigner-distribution functions, and the fractional Fourier transform," Appl. Opt. 33, 6188-6193 (1994).

11. A. W. Lohmann and B. H. Soffer, "Relationship between two transforms: Radon-Wigner and fractional Fourier," in Annual Meeting, Vol. 16 of 1993 OSA Technical Digest Series (Optical Society of America, Washington, D.C., 1993), p. 109.

12. D. Mendlovic, H. M. Ozaktas, and A. W. Lohmann, "Self Fourier functions and fractional Fourier transforms," Opt. Commun. 105, 36-38 (1994). 
13. R. G. Dorsch, A. W. Lohmann, Y. Bitran, D. Mendlovic, and H. M. Ozaktas, "Chirp filtering in the fractional Fourier domain,” Appl. Opt. 11, 7599-7602 (1994).

14. H. M. Ozaktas and D. Mendlovic, "Fractional Fourier transform as a tool for analyzing beam propagation and spherical mirror resonators," Opt. Lett. 19, 1678-1680.

15. B. E. A. Saleh and M. C. Teich, Fundamentals of Photonics (Wiley, New York, 1991).

16. A similar limiting process is discussed in P. J. Readon and R. A. Chipman, "Maximum power of refractive lenses: a fundamental limit," Opt. Lett. 15, 1409-1411 (1990).

17. M. J. Bastiaans, "The Wigner distribution applied to optical signals and systems," Opt. Commun. 25, 26-30 (1978).

18. M. J. Bastiaans, "Wigner distribution function and its application to first-order optics," J. Opt. Soc. Am. A 69, $1710-1716$ (1979).
19. M. J. Bastiaans, "The Wigner distribution function and Hamilton's characteristics of a geometric-optical system," Opt. Commun. 30, 321-326 (1979).

20. M. J. Bastiaans, "Propagation laws for the second-order moments of the Wigner distribution function in first-order optical systems," Optik 82, 173-181 (1989).

21. M. J. Bastiaans, "Second-order moments of the Wigner distribution function in first-order optical systems," Optik 88, 163-168 (1991).

22. L. Onural, "Diffraction from a wavelet point of view," Opt. Lett. 18, 846-848 (1993)

23. P. Pellat-Finet, "Fresnel diffraction and the fractional-order Fourier transform," Opt. Lett. 19, 1388-1390 (1994).

24. P. Pellat-Finet and G. Bonnet, "Fractional order Fourier transform and Fourier optics," Opt. Commun. 111, 141154 (1994). 